\title{
Dynamic perceptual mapping
}

\author{
Michael Greenacre \\ Department of Economics and Business \\ Universitat Pompeu Fabra
}

Ramon Trias Fargas, 25-27

08005 Barcelona

SPAIN

E-mail: michael@upf.es

\begin{abstract}
Perceptual maps have been used for decades by market researchers to illuminate them about the similarity between brands in terms of a set of attributes, to position consumers relative to brands in terms of their preferences, or to study how demographic and psychometric variables relate to consumer choice. Invariably these maps are two-dimensional and static. As we enter the era of electronic publishing, the possibilities for dynamic graphics are opening up. We demonstrate the usefulness of introducing motion into perceptual maps through four examples. The first example shows how a perceptual map can be viewed in three dimensions, and the second one moves between two analyses of the data that were collected according to different protocols. In a third example we move from the best view of the data at the individual level to one which focuses on between-group differences in aggregated data. A final example considers the case when several demographic variables or market segments are available for each respondent, showing an animation with increasingly detailed demographic comparisons. These examples of dynamic maps use several data sets from marketing and social science research.
\end{abstract}

Keywords: Animation, brand-attribute maps, correspondence analysis, multidimensional scaling, perceptual map, visualization.

\section{NOTE TO READERS}

This article contains dynamic graphics embedded into the PDF file. You need the latest version of Acrobat reader (version 9.0) to be able to see these animations. You can also download the videos in a powerpoint presentation by clicking on this link (or copying it to your browser)

www.econ.upf.edu/ michael/DynamicPerceptualMapping.pps 


\section{Introduction}

Perceptual mapping is the application of multidimensional scaling (MDS) and various factorial techniques, such as principal component analysis (PCA), correspondence analysis (CA) and discriminant analysis (DA), to data that reflect consumer perceptions of brands in the marketplace (for a comprehensive account of MDS, see Borg and Groenen (2005); for a recent account of correspondence analysis and related methods, see the multi-authored book edited by Greenacre and Blasius (2006), and Greenacre (2007); for an account of perceptual mapping in marketing, see Green and Wind (2004)). In this context a perceptual map is a graphical display in which brands are depicted in such a way that distances between brands reflect their differences as measured by the variables on which they are evaluated. These variables can be as simple as rank-orderings provided by a sample of consumers, or as detailed as a set of attributes for which each consumer expresses their relationship with the brands, either in the form of an indication of brand-attribute associations or rating-scale measurements. In marketing research as well as the broader field of social science in general, a common issue is the relationship between demographic variables and the answers to a set of substantive questions, and perceptual maps can provide easy-to-digest visualizations of these relationships, where the map is designed to maximize the "explained variance" in the data, either at the individual or at the aggregate level.

Invariably, owing to the nature of print media (in the case of research articles and technical reports) and slide shows (in the case of presentations at conferences and meetings), perceptual maps are static and two-dimensional. The following quotation is a true reflection of the present state of affairs: "Perceptual maps can have any number of dimensions but the most common is two dimensions. Any more is a challenge to draw and confusing to interpret." (http://en.wikipedia.org/wiki/Perceptual_mapping). However, there are rapid changes taking place both in the world of graphics as well as in the publishing world: in particular, dynamic graphics is becoming easy and cheap, while publishing is becoming increasingly electronic and affording 
new opportunities for publishing video material online. In this article I will demonstrate the advantages, sometimes quite astounding, of this new technology.

The idea of introducing motion into graphics can be exploited in very many different ways, and here we shall restrict ourselves to four applications: (1) extending perceptual maps to show a third dimension; (2) showing the difference between maps of data collected under different protocols; (3) showing how maps can dynamically illustrate differences between individual- and group-level analyses and (4) investigating relationships between substantive variables and demographic characteristics at an increasing level of detail. Each application will be illustrated using real marketing or social science data.

\section{Into the third dimension}

Viewing data in three dimensions is not unusual, but publishing maps that can be truly interpreted in three dimensions is. Several computer packages allow visualizing points in three dimensions and rotating them in real time, either using the keyboard arrow keys or the mouse, for example XLSTAT's 3d-miner (Addinsoft, 2007) or the rgl package in R (R Development Core Team, 2007). The additional information provided by a perceptual map's third dimension can be measured - this improvement is, by definition, less than the information conveyed on the first or second dimensions but it is nevertheless positive, and can make a difference to the interpretation, as we show in this example. Consider Figure 1, the usual two-dimensional map of a typical brand-attribute data set, published by Torres and Bijmolt (2008). The data are the associations between a set of 10 deodorant brands and a set of 11 attributes, expressed by a representative sample of 198 consumers. The perceptual map is the so-called symmetric map

\footnotetext{
* A proposal to the Elsevier Grand Challenge about dynamic graphics in the life sciences, submitted by Greenacre and Hastie (2008), made it to the semi-finals of this worldwide competition, which had as its objectives "to improve the way scientific information is communicated and used" and "to generate useful new ideas that could have a widespread impact on scientific publishing in general." See www.elseviergrandchallenge.com.
} 
obtained using CA (for the software in R, see Nenadić and Greenacre (2007)), where brands and attributes are scaled in the same way. The map explains a respectable $77.4 \%$ of the data variance (called inertia in CA), and shows a contrast on the first axis between deodorants associated with the aesthetic fragrance attributes on the right, opposed to the more pragmatic attributes of "keeping dry", "preventing odour" and "no irritation", on the left. Correspondingly we have the brand Mum well distinguished on the right opposed to several brands such as Soft \& Gentle, Impulse and Right Guard on the left. On the second axis we see the brand Natrel Plus well separated from the others in the same direction as the attribute "costs less". The spatial interpretation is quite clear and this might possibly be the only perceptual map studied by the researchers. They would conclude that the brand Body Shop, which is close to the middle of the map (that is, the point that represents the average brand and average attribute) has no distinctive features relative to the other brands.

\section{Insert Figures 1 and 2 about here}

\section{(refer to the dynamic Figure 2 in the additional presentation file)}

Figure 2 is the perceptual map in three dimensions, and will rotate in the online version.

Rotation takes place around the first axis and one immediately gets a different impression of the configuration. In particular, as the display is rotated the brand Body Shop becomes more and more separated from the other brands, showing that it is indeed not as similar to the others, nor as close to the average, as thought previously. The brand Sure which is also close to the center in Figure 1, does not change its distance to the center during the rotation, showing this brand to be much closer to average than Body Shop. In three dimensions we are now accounting for $87.0 \%$ of the inertia and thus obtaining a view of the data that is closer to reality. In summary, by introducing the third dimension and increasing the amount of information displayed, we have realized that Body Shop is a fairly isolated deodorant brand, not at all an average one. However, this conclusion should be judged in the light of the fact that Body Shop is the least evoked brand, 
thus has the least associations and a position in the map with the largest uncertainty (see Torres and Bijmolt, 2008).

\section{Moving between two matched data sets}

The data set analyzed in Figures 1 and 2 was obtained by first establishing for each respondent, in the sample of 198 consumers, a personal list of evoked brands, and then asking the respondents to select from a list of attributes those that they felt were strongly associated with each of their evoked brands. In the same study by Torres and Bijmolt (2008), another sample of 203 consumers is analyzed, where now each person was asked to consider the attributes one at a time and then select the brands from their evoked set that associated strongly with the particular attribute. The first table is called the brand-to-attribute associations matrix and the second the attribute-to-brand associations matrix - both have the same rows and columns, but the data have been collected according to different protocols. To study the differences between the results, the above authors consider a method for analyzing so-called "matched matrices", proposed by Greenacre (2003). As an alternative approach, motion can be used to move from the analysis of the first table to that of the second so that differences in the results induced by the two data collection methods can be observed in real time.

In order to compute the frames for this dynamic mapping, suppose the first data set is denoted by $\mathbf{N}_{1}$ and the second by $\mathbf{N}_{2}$, and that they are matched in the sense that they both have exactly the same rows and columns and data measured on the same scale (all counts in this case). We define a hybrid of the two matrices by the convex linear combination:

$$
\mathbf{N}=\beta \mathbf{N}_{1}+(1-\beta) \mathbf{N}_{2}
$$

and analyze the matrix $\mathbf{N}$ as $\beta$ is reduced from 1 to 0 . The first analysis (for $\beta=1$ ) is of the brands-to-attribute associations and the last one (for $\beta=0$ ) is of the attributes-to-brands 
associations, while all intermediate values of the mixing parameter ¡Error! Vínculo no válido. yield analyses between these two extremes. In practice we consider ¡Error! Vínculo no válido. $=1,0.99$, $0.98, \ldots, 0.02,0.01,0$, and each analysis of $\mathbf{N}$ provides a frame for a 101-frame animation.

Since $\mathbf{N}_{1}$ and $\mathbf{N}_{2}$ are matrices of frequencies we can apply CA to each hybrid matrix $\mathbf{N}$. Care has to be taken that the solution does not reflect on the principal axes, so two stable points are selected along the two principal axes and their signs are maintained constant across the maps (in this example, the deodorant Mum is always positive on the first principal axis, and Natrel Plus always negative on the second)..$^{\dagger}$ Figure 3 shows the result - in the static figure we just show the final map of the attributes-to-brands matrix, while in the dynamic version the movement of the brands and attributes allows us to discern the essential differences between the two data collection methods. For example, whereas Natrel Plus was quite isolated in its association with the attribute "costs less" in the brands-to-attributes analysis, it is joined by Body Shop (and to a certain extent Sure and Secret as well) in the attributes-to-brands analysis. By contrast, brands such as Mum and Right Guard remain stable across the animation.

\section{Insert Figure 3 about here \\ (refer to the dynamic Figure 3 in the additional presentation file)}

Additional diagnostics are given in the dynamic version of Figure 3. In the lower right hand box the upper curve (moving from right to left as $\beta$ decreases) traces out the total inertia of the hybrid analysis, the curve below depicts the part of that inertia explained by the two-dimensional map, and the lower pair of curves shows the individual parts of inertia on the two principal axes. The percentage of inertia is shown numerically and is seen to increase up to point and then descend again. In the left hand box (moving from left to right as $\beta$ decreases) the Procrustes

\footnotetext{
${ }^{\dagger}$ Rotations in the map are more problematic, as they can involve axis switches, but we have developed an algorithm to identify this situation and to make the appropriate adjustments in the signs of the coordinates. Rotations are thus seen smoothly if a "stable" point crosses an axis - Figure 7 is an example of this.
} 
statistics for the attributes (in red) and the deodorant brands (in blue) are traced out (Gower and Dijksterhuis, 2004). This statistic measures how far away the display in the present frame is from the initial one - hence, as can be seen during the animation, the brand points change their positions more, with a Procrustes of $36.4 \%$ between the last and the first frames, compared to $17.2 \%$ for the attributes.

\section{From individual to aggregated data}

In many studies the original respondent-level data are of interest as well as the segmentation of the respondents into groups, which are either pre-defined demographic groups or clusters that have been identified in a separate exercise. So we have two alternative levels of investigation of the data set: individual respondent level, and aggregated group level, and a perceptual map is possible at either level. But what is the difference between the two maps, and which variables are important in the individual-level analysis compared to the group-level one? A dynamic perceptual map can answer this question by moving smoothly between the two levels of analysis. We shall describe how this is done in the case of $\mathrm{CA}$, but the principle is the same for PCA or any MDS technique that can handle individual and aggregated data.

The data set consists of the responses of 5,934 people in three selected countries, USA, Spain and Russia, in the International Social Survey Programme's survey of Family and Changing Gender Roles in 1994 (ISSP, 1994), data that have already been analyzed "statically" by Greenacre and Pardo (2006) and Greenacre (2007: chap. 17). We consider four questions that ask whether married women should work or stay at home, at four consecutive time-points in their married lives: (1) before having a child, (2) with a preschool child, (3) when the youngest child is at school, and (4) when the children have left home. The possible responses in each case are "work full-time", "work part-time", "stay at home", or several types of non-substantive response that are grouped together, "unsure/don't know/non-response". In addition, we have 
data on several demographic variables for each respondent: country, gender, age, education, and so on. On the one hand, we could do a multiple correspondence analysis (MCA) of the respondent-level data (see, for example, Hoffman and Franke (1986) or, for an updated recent account, Greenacre $(2006,2007))$, while on the other hand we could do a simple CA of the cross-tabulations of one or more demographic variables with the question responses. These two analyses represent two extremes between which we can define a continuous set of analyses, similar to the idea in the previous section.

In MCA, data at the respondent level are coded as dummy variables in a respondents-bycategories indicator matrix, with 5,934 rows and 16 columns generated by the dummy variables from the four questions each with four possible responses. MCA is the CA of this large indicator matrix, resulting in a perceptual map of 16 category points, but also points for the 5,934 respondents. We are usually not interested in the positions of individual respondents, especially in the case of this huge data set, but rather in the positions of groups of respondents, for example, the average of the respondents in each of the countries. These averages can be displayed in MCA as so-called supplementary points, which are defined by additional rows aggregating the respondents into the three country groups. The data structure is shown in Figure 4. Figure 5 shows the MCA map of the response and demographic categories, depicted by their labels, and the respondent points as dots (we do not see 5934 different points here because many response patterns are identical and thus pile up at the same locations). The question response categories show a clear separation horizontally of the missing responses (on the left) from the substantive responses (on the right), and a vertical spread of the substantive categories going from a liberal attitude at the bottom (for example, $\mathbf{2} \boldsymbol{W}$ : women should work fulltime even when they have an infant child) to a traditional attitude at the top (for example, $\mathbf{1 H}$ : women should stay at home even before they have their first child). The demographic categories form a blur in the centre of the display and are poorly separated in this map, since the map has been constructed to 
separate out the response categories as much as possible in terms of their respondent-level associations. Since there are many respondents giving several missing responses to these four questions, these categories are highly associated and cause their large separation on the first principal axis, which is the dominant feature of the map.

\section{Insert Figures 4 and 5 about here}

Another way of thinking of Figure 5 is as follows. Consider scattering the respondents on a plane, with the constraint that the variance of these points along the two coordinate axes is equal to 1 . Then compute the average positions of the groups of respondents that fall into each of the response categories, and the average positions of those that fall into each demographic category. Now solve the following problem: what configuration of the respondent points will maximize the spread of the question response categories? This is the MCA solution of Figure 4, alternatively called "optimal scaling" or "homogeneity analysis" (Gifi (1990), Michailidis and de Leeuw (1998)) and it is aimed on showing maximum variance in the response categories. The depiction of the demographic groups as average points is not optimized at all in this map, and so it is not surprising that we see such small differences between the countries, genders or age groups. Figure 6(a) shows the category points of Figure 5 without the respondent points, where the scale of the map is amplified so that the demographic groups are spread out more.

\section{Insert Figures 6(a) and (b) about here}

At the other extreme, the CA of the data aggregated by country is the CA of the cross-tabulations that are appended onto the indicator matrix in Figure 4. If one really wanted to see all the individual respondent points, one could display them as supplementary points in the space of the aggregated country averages, but as before we are interested in their positions mainly to compute the average positions of response category or demographic groups. Figure $6(\mathrm{~b})$ shows the 
positions of the category and demographic averages now that the analysis is specifically focused on separating the demographic groups. The substantive opinion gradient is now horizontal, from traditional on the right to liberal on the left, forming an arch, which is a well-known phenomenon in CA. The countries, genders and age groups are now more clearly separated, with the USA on the liberal side, Russia on the traditional side. Spain lies positive on the vertical axis within the arch, demonstrating that Spain is polarized on this issue, with more than average liberal and traditional responses, and fewer of the compromise responses such as the part-time option, which is lower down vertically. The age groups are spread out in the order we would expect, forming an arch from young on the liberal side to old on the conservative traditional side. Male responses tend to the traditional side of the average and females to the liberal side, again as one would expect.

In both the MCA of the respondent level indicator matrix $\mathbf{Z}$ and the CA of the aggregated matrix $\mathbf{N}$, it is the matrix of profiles that is analyzed, the rows relative to their row totals. In the MCA of $\mathbf{Z}$ each respondent receives an equal weight $(1 / n)$, but could just as well receive a differential weight $w_{i}$ if, for example, there was a need to compensate for some lack of representativeness in the sampling. In either case the matrix $\mathbf{N}$ has row weights equal to the sum of the weights of respondents in each group - in the usual equally weighted case, $\mathbf{N}$ will have weights proportional to the number of respondents in each group. The two extreme forms of analysis, $\mathrm{CA}$ of $\mathbf{Z}$ (i.e., MCA) and CA of $\mathbf{N}$, can be linked by transferring weight gradually from the individual points to their averages, or centroids. Shifting the weight from $\mathbf{Z}$ to $\mathbf{N}$ can be engendered by the following convex combination, in the same spirit as (1):

$$
\mathbf{m}(\beta)=\beta\left[\begin{array}{l}
\mathbf{w} \\
\mathbf{0}
\end{array}\right]+(1-\beta)\left[\begin{array}{l}
\mathbf{0} \\
\mathbf{r}
\end{array}\right]
$$

The mixing parameter $\beta$ is varied smoothly from 1 to $0-$ at the start $(\beta=1)$ the analysis is of $\mathbf{Z}$ only, with no weight on $\mathbf{N}$, so that the demographic groups are supplementary points on the map, 
as in Figure 5 or Figure 6(a); while at the end $(\beta=0)$ the analysis is of $\mathbf{N}$ only, i.e. the centroids, as in Figure 6(b). In the intermediate stages all points, individuals and centroids, have some positive weight and the whole augmented matrix is analyzed.

Figure 7 is the dynamic perceptual map which shows the smooth transition, as $\beta$ descends from 1 to 0 , from the individual-level analysis to the aggregate-level one. The box at lower right, which moves from right to left as $\beta$ descends, shows the total inertia of the demographic categories as a constant value at the top. The next curve is the part of this inertia explained by the map at any given moment - as $\beta$ decreases this part of inertia is seen to increase as more between-group inertia is being explained. The two lowest curves show the respective parts of inertia on the two principal axes (notice how these cross over when the display starts to rotate). The box on the left shows the evolution of the two Procrustes statistics for the respondent points and demographic points compared to the initial individual-level analysis, moving from left to right as $\beta$ descends. Here one can see that up to near the end the configurations are pretty stable, just rotating, but then as all the mass is finally transferred to the demographic centroids the map changes dramatically, as measured by the Procrustes statistics which ascend rapidly. We see that, as we move to the map of the aggregated data, the missing value categories move towards the center because they are not as important as in the individual-level map: they might occur in the same individuals, but at the demographic group level the association is weaker.

\author{
Insert Figure 7 about here
}

(refer to the dynamic Figure 7 in the additional presentation file)

\title{
Showing increasing detail in relationships with demographic variables
}

In Figure 6(b) and the dynamic perceptual map of Figure 7, we are focusing marginally on the inter-group structure between countries, between age groups and between sexes and not in 
combination (i.e., not showing interaction differences). An alternative map could show all $3 \times 6 \times 2=36$ country/age/sex combinations (called "interactive coding") and their associations with the response categories. Faced with these two possibilities, we can either make static displays of each one of them, or we can show dynamically what happens as we move from the less detailed analysis to the one with all the interactively coded demographics. There are various ways of constructing the dynamic map, and here we illustrate a simple "morphing" of the maps.

Given two maps we can morph them by making a linear interpolation between each point in the first map and its destination position, or positions in this case because the points are splitting. Figure 8 has been constructed in this way, first moving from a map which separates the three countries to an intermediate map which separates out the sexes in each country, so that each country is seen splitting into a male and female group. We can immediately see that the difference between the sexes is relatively small compared to the differences between the three countries, with USA showing the least difference. In Spain and the USA males are moving slightly to the right, the conservative side of the map, and the females to the left, the liberal side. The Russian male-female difference is vertical, which means that Russian males are just slightly more polarized. Then there is a second stage of the animation which separates each country/sex group into its set of age groups, thus terminating with all 36 combinations of the demographics. Here the Spanish male and female groups start to spread out widely from left (youngest groups) to right (oldest groups), especially the males. At the other extreme Russian males of different age groups differ only slightly from their average. Notice that in the middle and last frames of the animation, shown as the second and third maps in the static version of Figure 8, the average point of a set of category points is given in the previous map: for example, the six Spanish male points (ESM1 to ESM6) in the last frame have as their average the point ESM in the middle frame. The dynamic version of Figure 8 shows quite effectively the changes in the map induced by looking at such demographic interactions. 


\author{
Insert Figure 8 about here \\ (refer to the dynamic Figure 8 in the additional presentation file)
}

\title{
Discussion and conclusions
}

Four examples of dynamic perceptual mapping have been given, to demonstrate the ability of motion graphics to convey more understanding and a richer interpretation of marketing and social research data. There are many more possibilities, of which we list some that would be of interest to marketing researchers:

- Suppose that the same data have been recorded at two or more time points (these could be based on separate samples in a trend study, or the same respondent panel in a longitudinal study). Rather than produce separate perceptual maps, motion could be used to show a smooth transition between time periods by interpolation.

- Suppose that the map depends on an external variable; for example, in testing the effect of different durations of an advertisement, we have a matrix of data recorded for each time length. Motion can be used to show how perceptions change as the advertisement duration changes.

- Suppose that some of the data are missing, and that these missing values need to be imputed (i.e., estimated) before the map can be calculated. Usually there are several ways that imputation can be conducted, some more sophisticated than others; for example, from simple replacement by marginal averages to more complex schemes using multiple regression or finding clusters of respondents similar to those that have missing data. The influence of the imputation on the results can be investigated by allowing the missing values to vary according to the alternative schemes and observing how stable the map is. 
- Suppose there are two different ways of calculating the perceptual map; for example, there are two alternatives for defining inter-product distances based on multi-attribute data before the products are mapped. Motion can be used to show a smooth transition between the two potential results, to demonstrate the similarities and differences between the two approaches.

Dynamic graphics is not confined to perceptual maps and can be used fruitfully to animate time series data, bivariate plots that are conditioned on a third variable, and in data mining to conduct “guided tours" through data in multidimensional space (Greenacre and Hastie, 2008).

In his classic book on media aesthetics aimed at video and television production, Zettl (2005) lists the five principal aesthetic fields in visual communication: the first dimension of light/color; the two-dimensional field of area and screens within screens; the three-dimensional field of space; the four-dimensional field with time/motion; and the five-dimensional field with sound. We have demonstrated that our use of motion in perceptual mapping pushes us well along towards the fourth dimension, whereas scientific graphics is presently stuck in two dimensions. Adding sound would lead to a further improvement, and would complete the picture. One can realistically imagine a dynamic figure being accompanied by a sound bite by the author, explaining to the reader what is happening in the animation. All this is highly possible in the present-day trend towards online publishing. The challenge is to develop dynamic graphics which occupy the same amount of space as a traditional static figure in the online article, and occupy the reader for the same length of time, while increasing dramatically the data content that is communicated. Vision has been described as more of an intelligence than a sense, and our vision intelligence is clearly being under-utilized when it comes to showing numerical results as static figures in scientific publications - one just has to look at a photo for five seconds and then a five-second video of the same person to realize how much more can be assimilated and understood when motion is introduced. 
Tools need to be developed to allow users to produce dynamic graphics with the same facility as traditional static figures, for which many software options are available. All the dynamic perceptual maps in this article were produced using the freeware package $R$ ( $R$ core development team, 2007). Short programs were written to produce a sequence of frames, which were then packaged into an animated GIF file using Animation Shop by Corel (www.corel.com). Usually we have aimed at a 101-frame animation at approximately 20 frames per second, with a pause at the start and end, to give an approximate 10 -second animation. This animation can be produced to repeat two or three times, or to loop repeatedly until the reader decides to move on. Greenacre and Hastie (2008) provide an $R$ function to demonstrate how the task of the user can be simplified if the right software tools are developed. This development is an ongoing project of the present author. Finally, note that the latest version of Acrobat (Adobe, 2008) includes the possibility of embedding three-dimensional graphics and flash videos in PDF files, which is the alternative way we have produced the animations that are embedded in this working paper. 


\section{Acknowledgments}

This research has been encouraged and supported by the Fundación BBVA, Madrid, Spain, and the author expresses his gratitude to the Foundation's director, Prof. Rafael Pardo. The article was prepared during the author's sabbatical, spent partially at Stanford University where it benefited from discussions with Prof. Trevor Hastie. Thanks are due to the Universitat Pompeu Fabra for the granting of the sabbatical period, and partial support of Spanish Ministry of Education and Science grant MEC-SEJ2006-14098 is also acknowledged. Antonio Lirio of Abobe Systems Ibérica provided essential advice in the construction of the dynamic graphics.

\section{References}

Addinsoft (2007). XLSTAT. URL: http://www.xlstat.com.

Adobe (2008). Creative Suite 4. URL: http://www.adobe.com.

Borg, I. and Groenen, P.J.F. (2005). Modern Multidimensional Scaling, $2^{\text {nd }}$ Edition. New York: Springer.

Carroll, J.D., Arabie, P., Chaturvedi, A. and Hubert, L. (2004). Multidimensional scaling and clustering in marketing: Paul Green's role. In Green, P.E. and Wind, Y. (eds). Marketing Research and Modeling: Progress and Prospects, pp. 71-102. New York: Springer.

Gifi, A. (1990). Nonlinear Multivariate Analysis. Chichester, UK: Wiley.

Gower, J.C. and Dijksterhuis, G.B. (2004). Procrustes Problems. Oxford: Oxford University Press.

Greenacre, M. J., 2003. Singular value decomposition of matched matrices. Journal of Applied Statistics 30, 1101-1113.

Greenacre, M. J. (2006). From simple to multiple correspondence analysis. In Greenacre, M.J. \& Blasius, J. (eds.) Multiple Correspondence Analysis and Related Methods, Chapman \& Hall/CRC Press, London, pp. 41-76.

Greenacre, M. J. (2007). Correspondence Analysis in Practice. Second Edition. London:

Chapman \& Hall / CRC Press. Published in Spanish translation: La Práctica del Análisis de Correspondencias. Madrid: Fundación BBVA, 2008. Full text available online. 
Greenacre, M.J. and Blasius, J., eds (2006). Multiple Correspondence Analysis and Related Methods. London: Chapman \& Hall /CRC.

Greenacre, M.J. and Pardo, R. (2006). Subset correspondence analysis: visualization of selected response categories in a questionnaire survey. Sociological Methods and Research, 35, 193218.

Greenacre, M. and Hastie, T. (2008). Guided tours in $n$-dimensional space: dynamic visualization of multivariate data. Semi-final entry in the Elsevier Grand Challenge, MIT, Boston. http://www.econ.upf.edu/ michael/elsetwee.

Hoffman, D.L. and Franke, G.R. (1986). Correspondence analysis: graphical representation of categorical data in marketing research. Journal of Marketing Research, 23, 213 - 217.

ISSP (1994). Family and Changing Gender Roles II. International Social Survey Programme. URL: http://www.issp.org.

Michailidis, G. and de Leeuw, J. (1998). The Gifi system of descriptive multivariate analysis. Statistical Science, 13, 307-336.

Nenadić, O. and Greenacre, M. J. (2007). Correspondence analysis in R, with two- and threedimensional graphics: The ca package. Journal of Statistical Software, 20 (1).

URL http://www.jstatsoft.org/v20/i03/

R Development Core Team (2007). R: A Language and Environment for Statistical Computing. R Foundation for Statistical Computing, Vienna, Austria.

URL http://www.R-project.org

Torres, A. and Bijmolt, T.H.A. (2008). Assessing brand image through communalities and asymmetries in brand-to-attribute and attribute-to-brand associations. European Journal of Operational Research, 195, 628-640.

Zettl, H. (2005). Sight, Sound, Motion: Applied Media Aesthetics. 4th Edition. California: Wadsworth. 
Figure 1: Correspondence analysis of attribute-to-brand associations (Table 1 of Torres and Bijmolt (2008), with weighting of brands according to evoked frequency). Symmetric map with rows and columns in principal coordinates; total inertia = 0.05797; inertia explained in the two-dimensional map $=77.4 \%$.

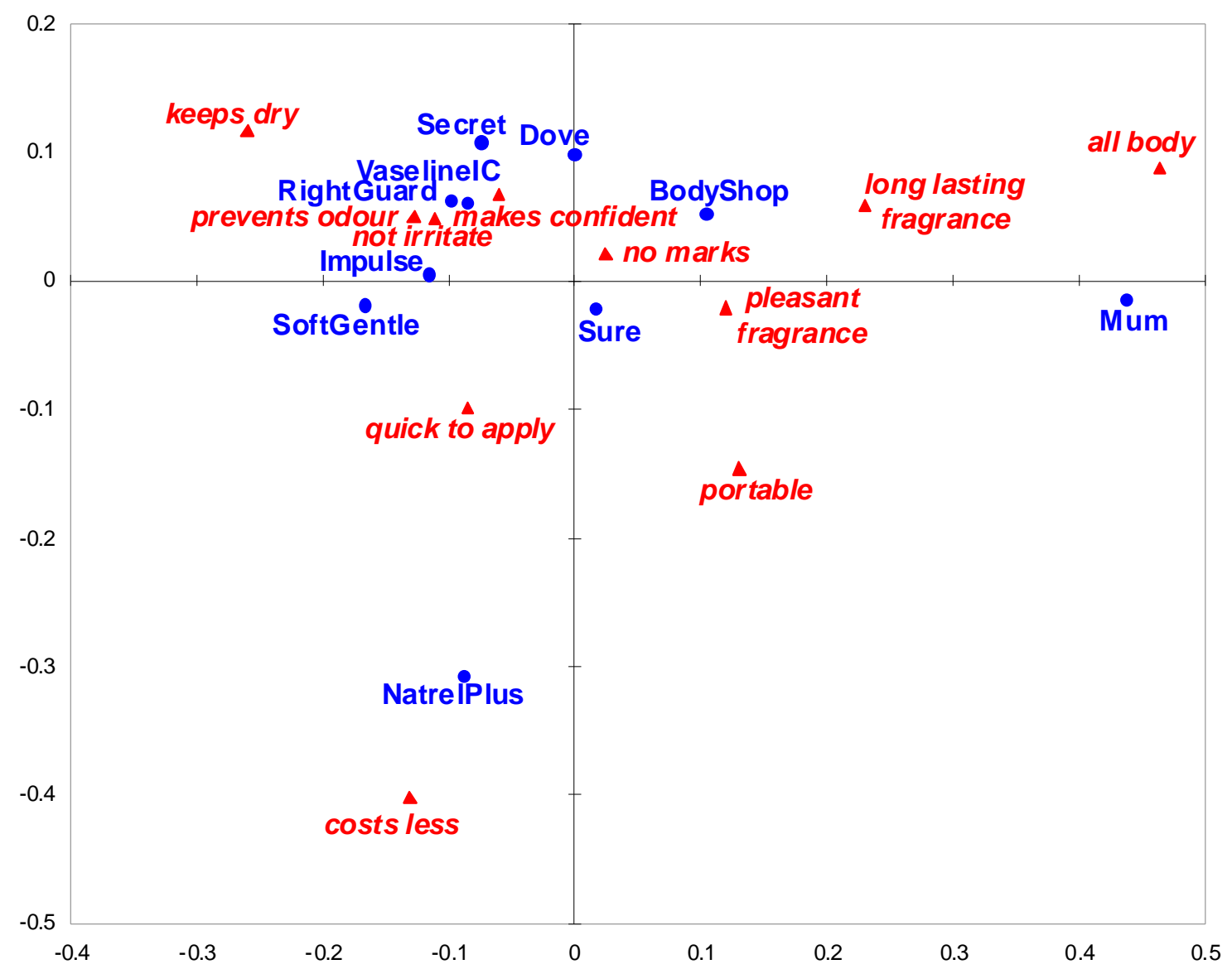


Figure 2: Same analysis as Figure 1, but shown in a three-dimensional rotation. An additional $9.6 \%$ of the inertia is now explained, showing that the brand Body Shop is actually isolated from the others. The three-dimensional perceptual map now explains $87.0 \%$ of the inertia. (In the dynamic version of this figure, shown on the following page, the rotation around the horizontal axis 1 will be shown as an animation; in the static version of this figure shown here, the image halfway through the rotation is shown, as dimension 3 comes into view).

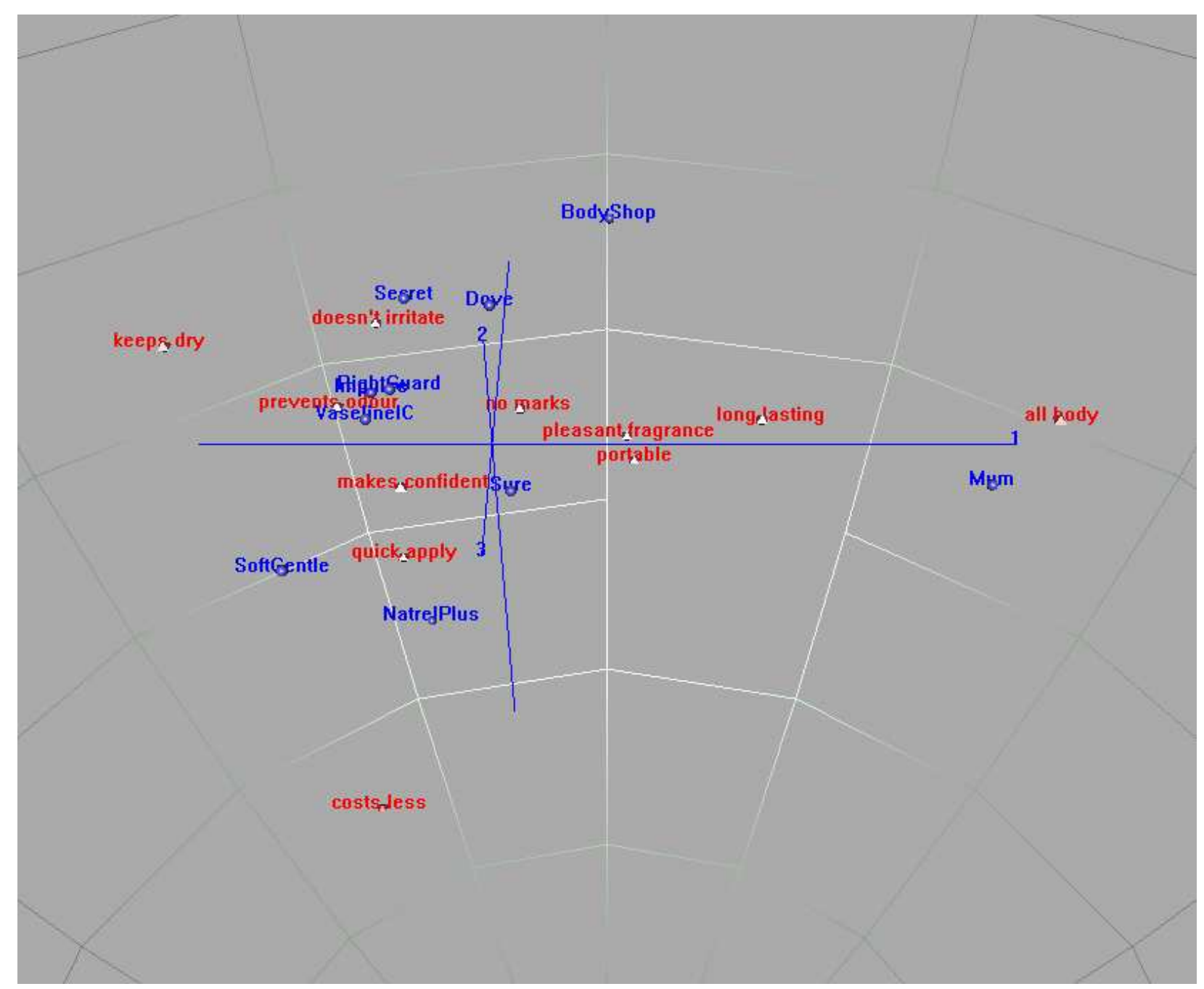


Figure 2 (dynamic version - click on image below): Same analysis as Figure 1, but shown in a three-dimensional rotation. An additional $9.6 \%$ of the inertia is now explained, showing that the brand Body Shop is actually isolated from the others. The three-dimensional perceptual map now explains $87.0 \%$ of the inertia. (In the dynamic version of this figure, shown on the following page, the rotation around the horizontal axis 1 will be shown as an animation; in the static version of this figure shown here, the image halfway through the rotation is shown, as dimension 3 comes into view).




Figure 3: Correspondence analysis of brand-to-attribute associations (Table 3 of Torres and Bijmolt (2008), with weighting of brands according to evoked frequency). Symmetric map with rows and columns in principal coordinates; total inertia $=$ 0.03874 ; inertia explained in the two-dimensional map $=79.5 \%$. Scale is identical to Figure 1, thus showing the reduction in dispersion of the points compared to the brands-to-attributes analysis. The dynamic version on the next page shows the smooth transition between the two analyses.

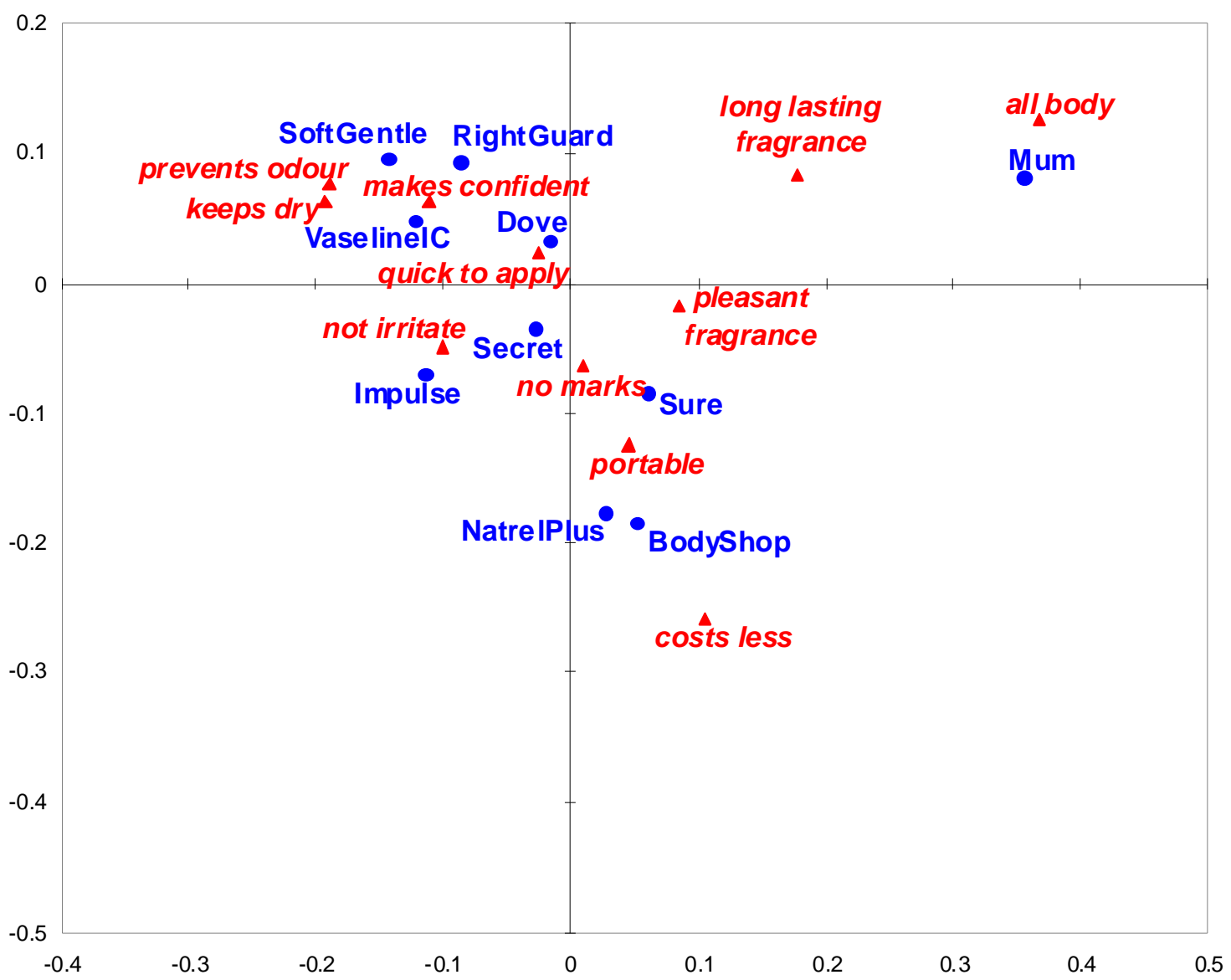


Figure 3 (dynamic version - click on image below): Dynamic transition between the analyses of attribute-to-brand associations (Figure 1) and brand-to-attribute associations (Figure 3, static version on previous page).

\section{From brand-to-attributes to attributes-to-brands}

Starts as CA map of brand-to-attribute associations...

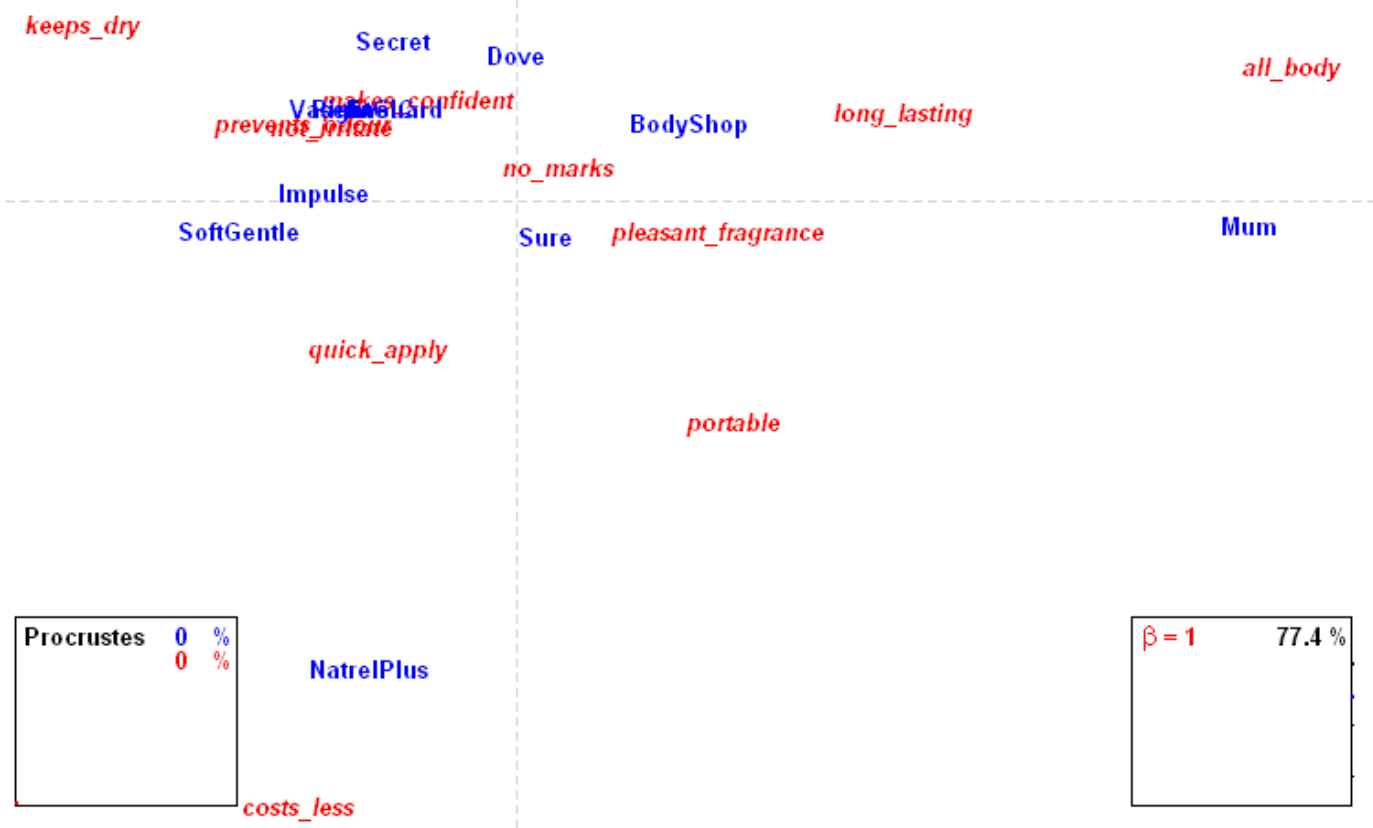


Figure 4: Data structure for individual respondent data $\mathbf{Z}$ and aggregated data $\mathbf{N}$. Each respondent is weighted equally by the inverse of the sample size $n$ or by a respondent weight $w_{i}$ which compensates for the representativeness of the respondent in his or her demographic group.

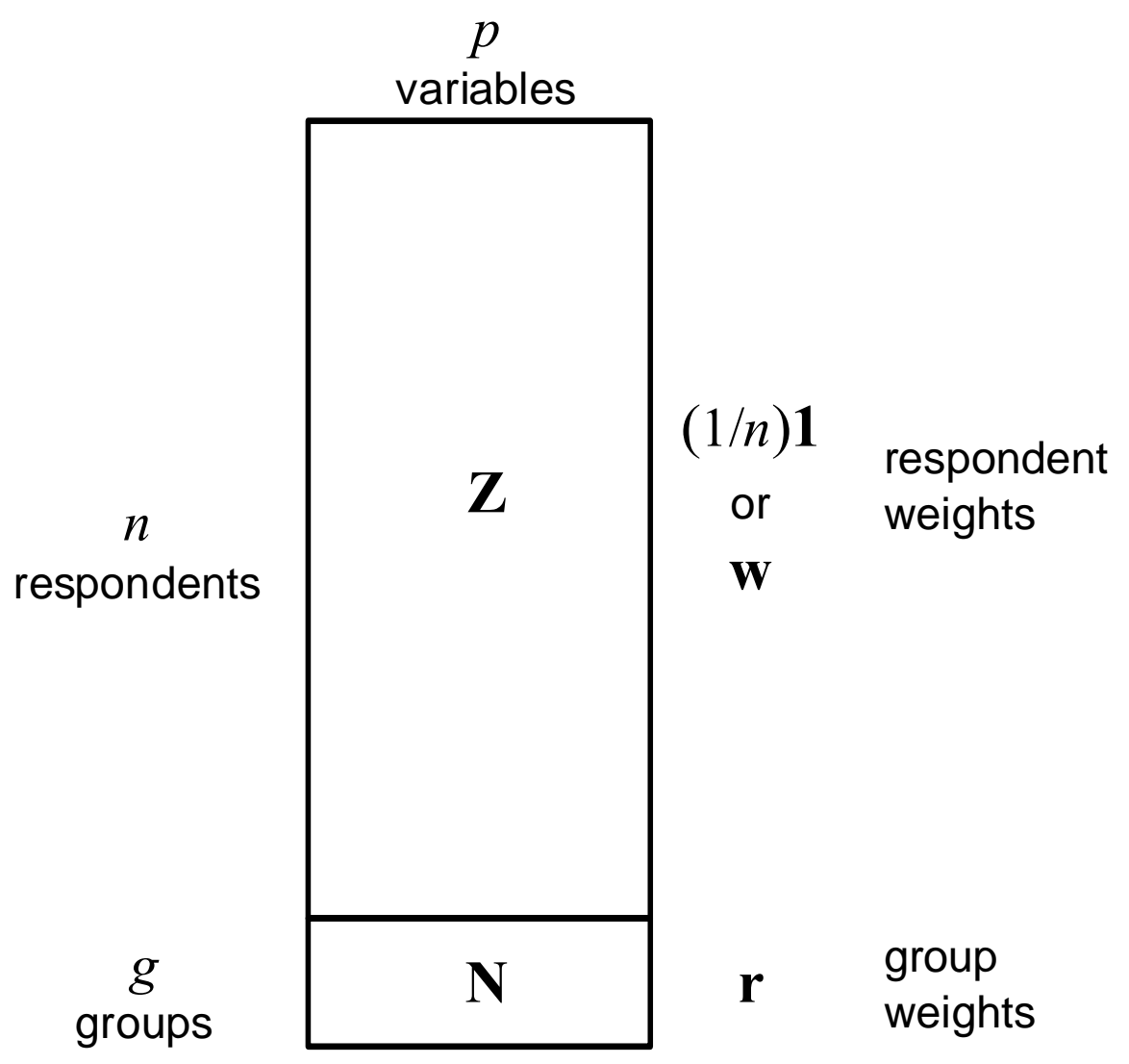


Figure 5: Multiple correspondence analysis (MCA) of individual respondent data (dots) showing response categories and supplementary demographic categories as average points. Figure 6(a) shows an enlargement of the category points only.

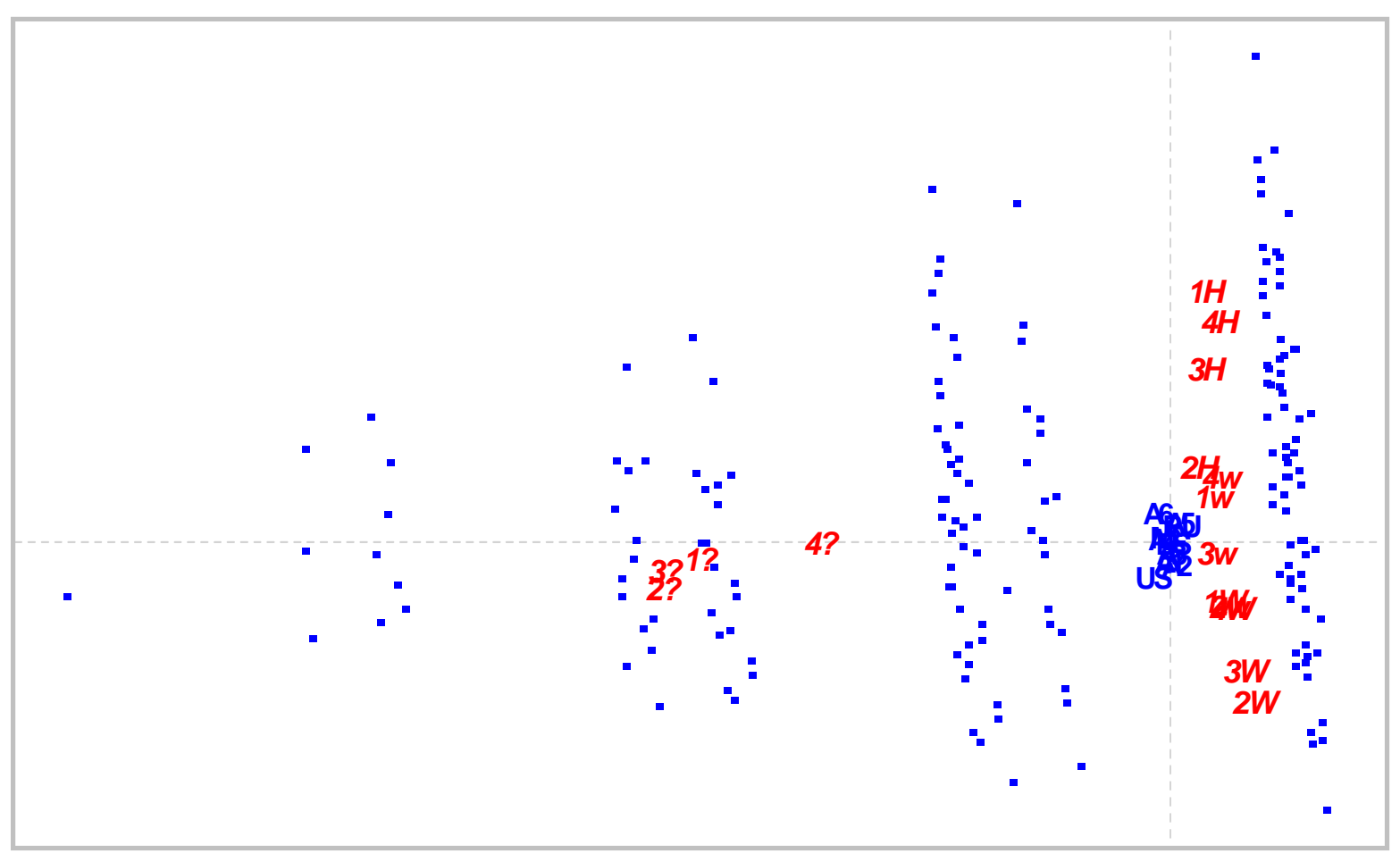


Figure 6: (a) Configuration of categories in Figure 5; (b) Configuration of categories in the correspondence analysis (CA) of cross-tabulations of demographic categories by response categories, i.e. the analysis of the aggregated data (the coordinates of all points in the latter map have been multiplied by 2 for legibility).

(a)

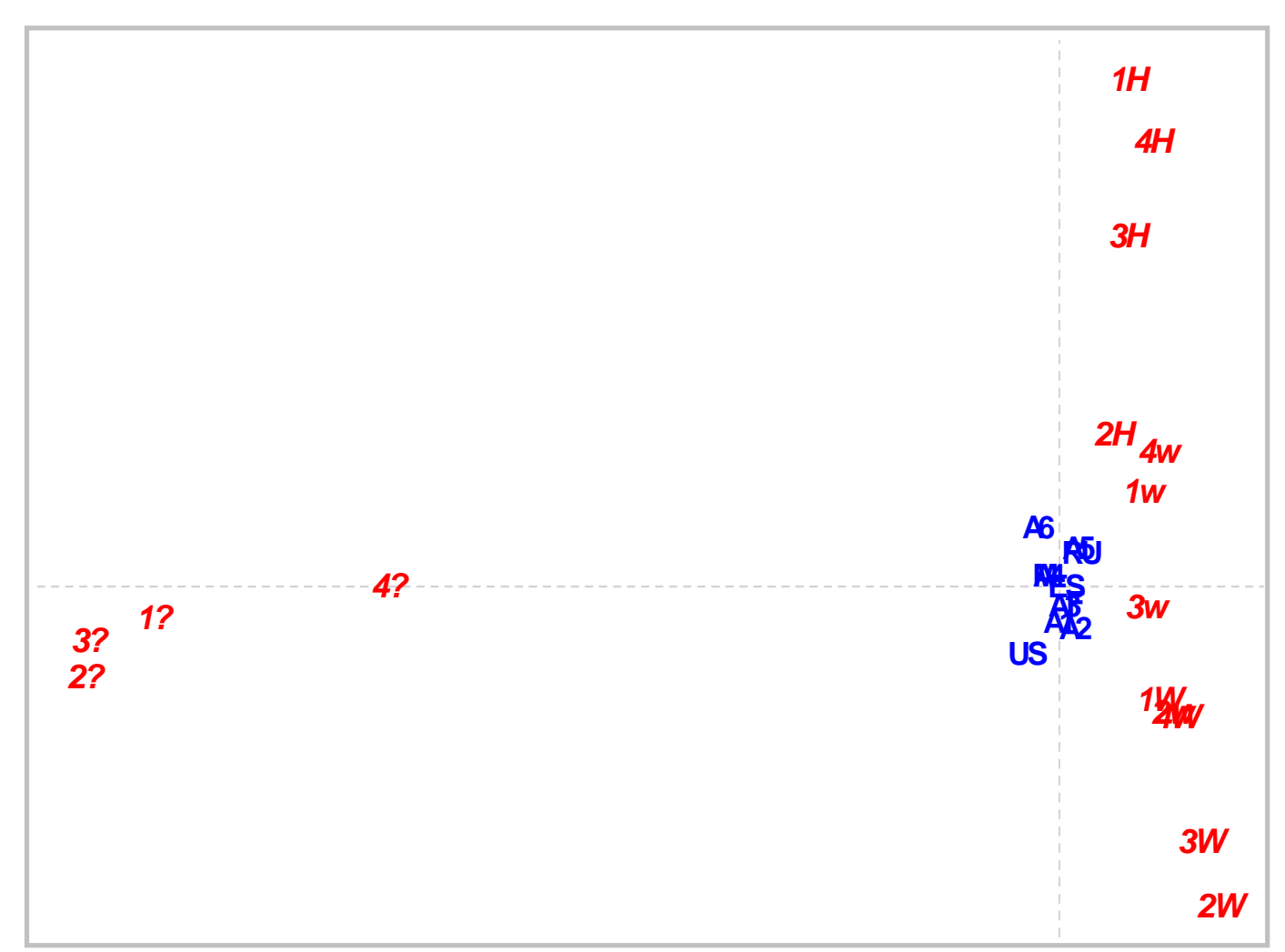

(b)




Figure 7: Single frame from the dynamic perceptual map moving from the individuallevel analysis to the aggregate-level analysis (aggregated into countries, genders and age groups). In the right hand box (which moves from right to left as $\beta$ decreases) the upper curve indicates the (constant) inertia of the demographic groups, the curve below shows the part of that inertia explained by the map (increasing as $\beta$ decreases), and the lower pair of curves shows the individual parts of inertia on the two principal axes. In the left hand box (which moves from left to right as $\beta$ decreases) the Procrustes statistics for the response categories, in red, and the demographic groups, in blue, are traced out. In this static frame, the image at $\beta=0.041$ is shown when the map is in the process of making the dramatic change.

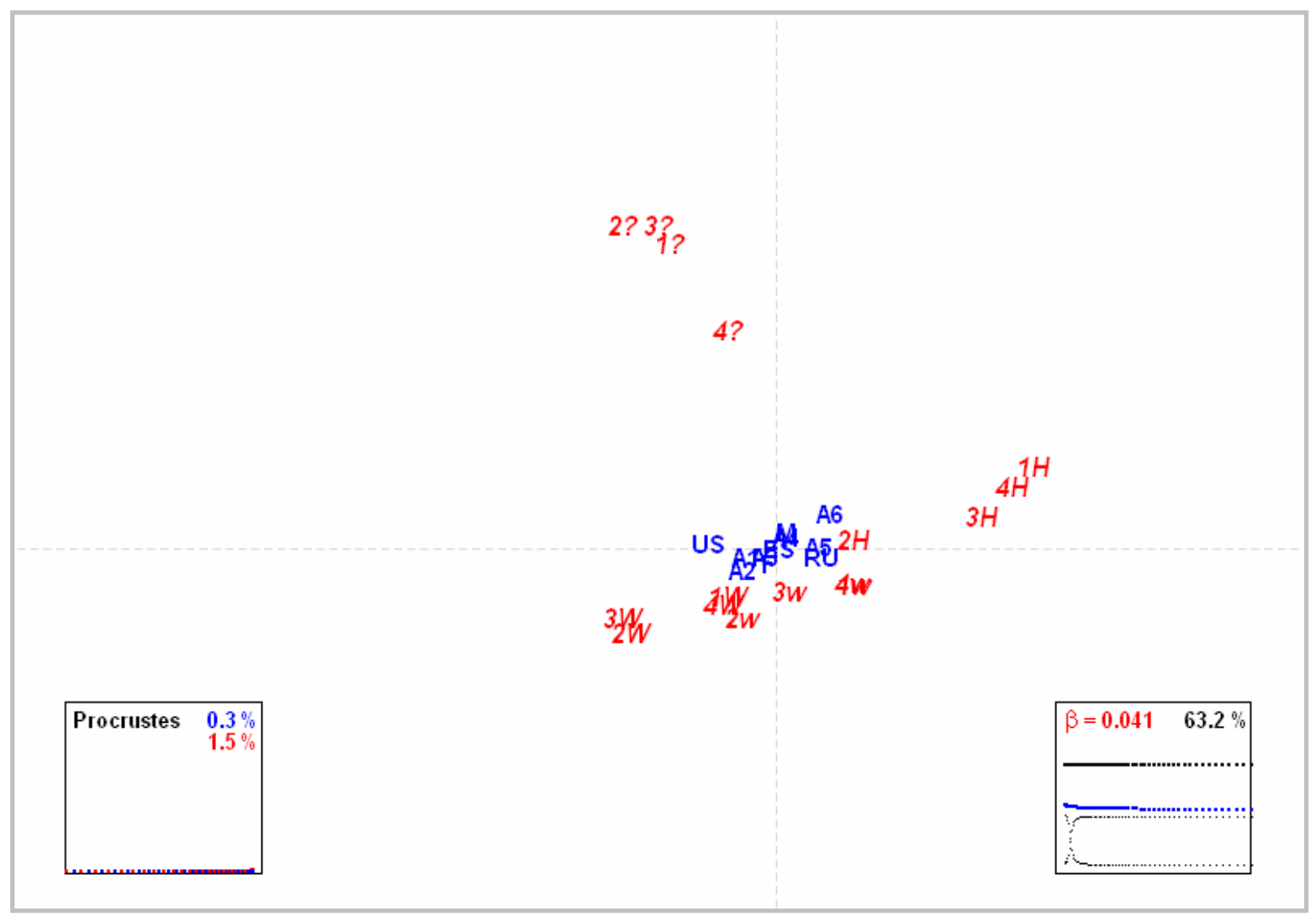

ES=Spain, RU=Russia, US=USA, M=male, F=female, $A 1=16-24$ years, $A 2=25-34, A 3=35-44, A 4=45-54, A 5=55-64, A 6=65+$ 
Figure 7 (dynamic version - click on image below): Dynamic perceptual map moving from the individual-level analysis to the aggregate-level analysis (aggregated into countries, genders and age groups). The mixing parameter $\beta$ is set to descend faster at the start when there is hardly any change, and slower at the end when change is rapid.

\section{From MCA of individuals to $\mathrm{CA}^{\mathrm{H}}$ of groups \\ Starts as MCA map with weight on ६̧ases...}

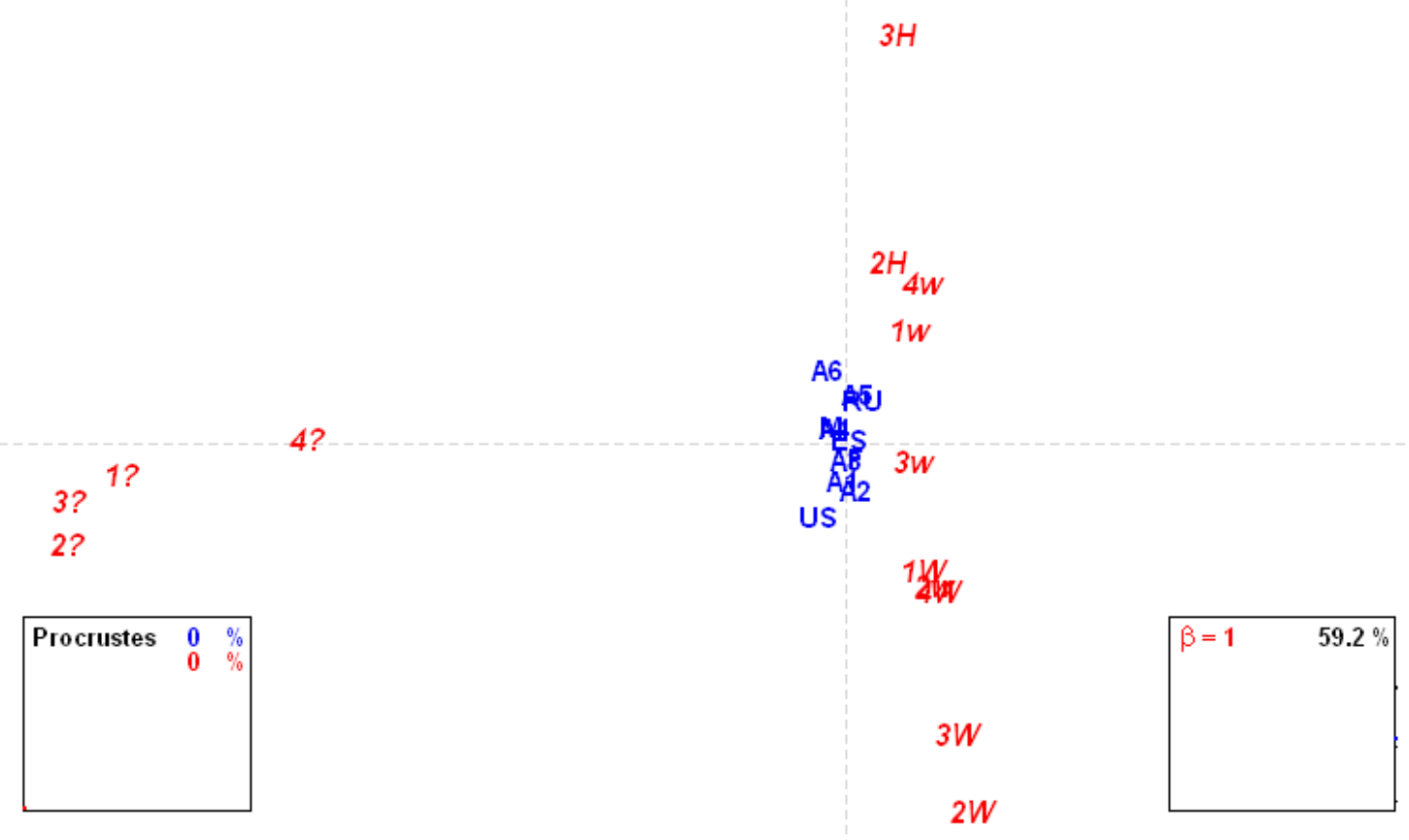


Figure 8: Images of the dynamic perceptual map moving from the representation of the country averages, splitting by sex, and then for each country-sex combination split into six age groups. In this static version we see the first frame (countries), the middle frame (countries $\times$ sex), and the last frame countries $\times$ sex $\times$ age).

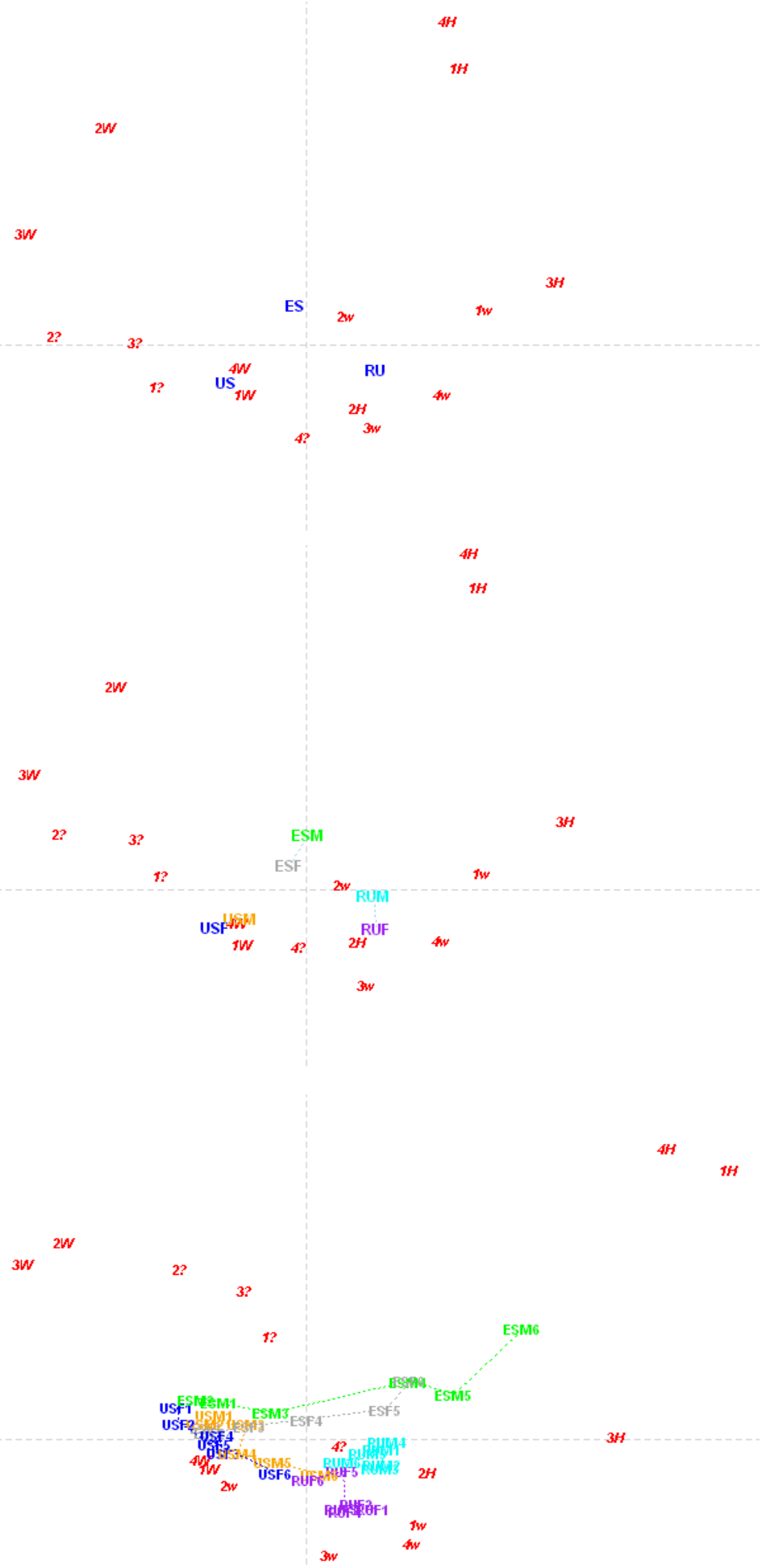


Figure 8 (dynamic version - click on image below): Dynamic perceptual map moving from the representation of the country averages, splitting by sex, and then for each country-sex combination split into six age groups.

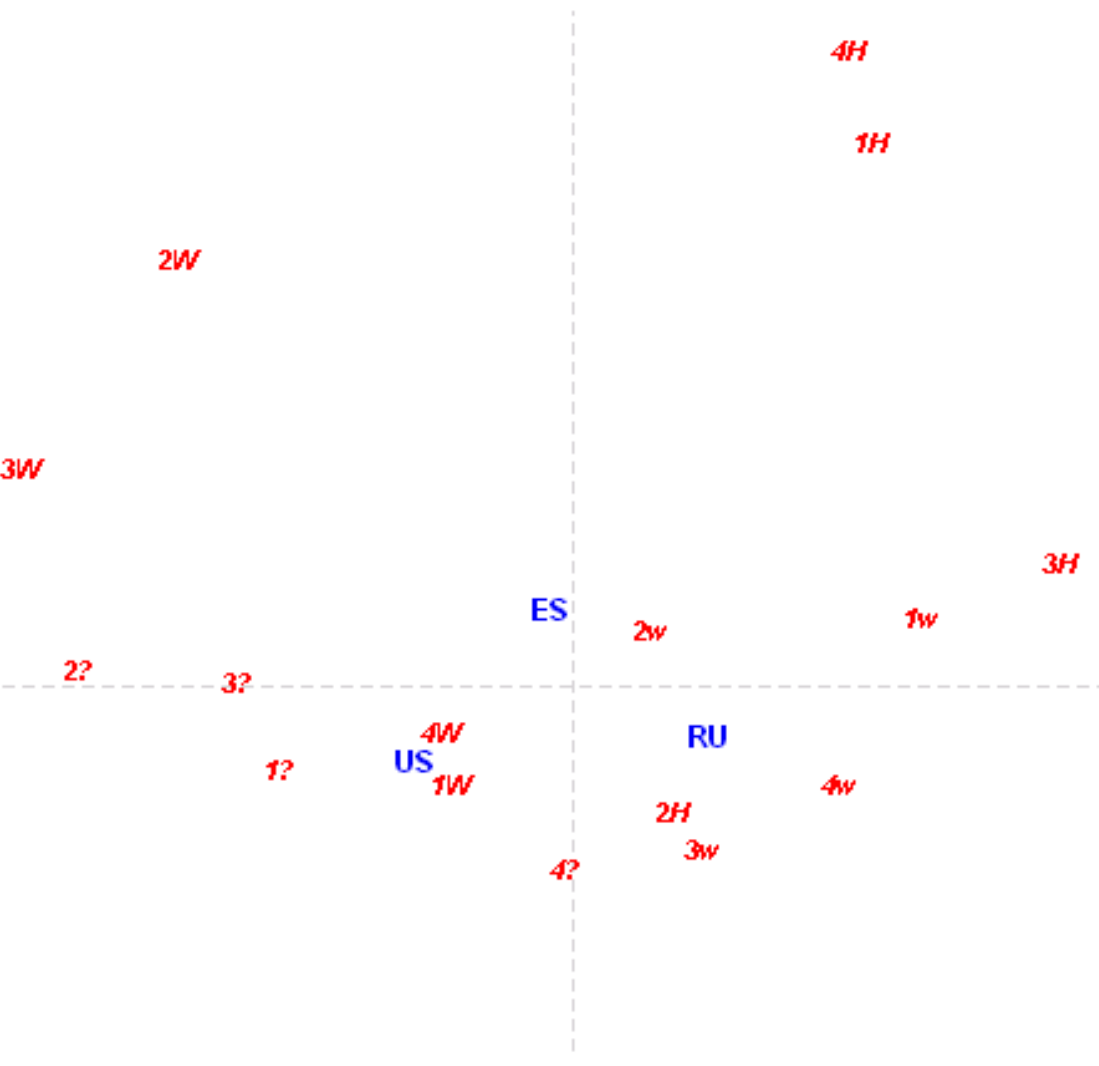

